\title{
Towards Autonomic Handover Decision Management in 4G Networks*
}

\author{
Joon-Myung Kang ${ }^{1}$, Hong-Taek $\mathrm{Ju}^{2}$, and James Won-Ki Hong ${ }^{1}$ \\ ${ }^{1}$ Dept. of Computer Science and Engineering, POSTECH, Korea \\ \{eliot, jwkhong\} @postech.ac.kr \\ ${ }^{2}$ Dept. of Computer Engineering, Keimyung University, Korea \\ juht@kmu.ac.kr
}

\begin{abstract}
Mobility management has become an important issue in 4G networks due to the integration of multiple network access technologies. Traditionally, only the received signal strength has been considered for the vertical handover. However, more considerations will be necessary to satisfy the end user's preferences. In this paper, we propose an Autonomic Handover Manager (AHM) based on the autonomic computing concept to decide the best network interface to handover in $4 \mathrm{G}$ networks. AHM decides the appropriate policy for the specific service or application without the user's intervention using the context information from the mobile terminal, the network and the user. We present the context information and the context evaluation function to decide handover based on the user preferences. We then describe the scenario to validate its feasibility using multimedia conferencing service on the mobile terminal.
\end{abstract}

Keywords: Autonomic Management, 4G Networks, Context Management, Vertical Handover, Mobility Management.

\section{Introduction}

Recently, the network trend has been moving towards the architectures that support wireless technologies, mobile users, multiple radio access technologies, heterogeneous networks and network convergence. In $4 \mathrm{G}$ networks, the users are in need of services using the terminal that supports multi-mode and multi-access, which means that the terminal can use any network regardless of network access technologies (e.g., CDMA, WLAN, Bluetooth and WiBro) or network providers. As a result, an interesting problem has surfaced on deciding the "best" network interface to use at any given moment. It is apparent that the decision should be based on various considerations such as the capacity of each network link, usage charge of each network connection, power consumption of each network interface, battery status of the mobile device and user preferences. They are called as context information from the network, the system and the user profile.

\footnotetext{
* This research was supported by the MIC (Ministry of Information and Communication), Korea, under the ITRC (Information Technology Research Center) support program supervised by the IITA (Institute of Information Technology Assessment)" (IITA-2005C1090-0501-0018) and by the Electrical and Computer Engineering Division at POSTECH under the BK21 program of the Ministry of Education, Korea.
} 
In this research area, mobility management which supports an efficient vertical handover [1], and Always Best Connected (ABC) [2] which provides the seamless service to the user are important aspects in the heterogeneous network environment. Horizontal handover is the process of maintaining the mobile user's active connections as it moves within the wireless network. Vertical handover, or intersystem handover, deals with the handover between different types of networks. Traditionally, research on the handover has been based on an evaluation of the received signal strength (RSS) at the mobile node. However, traditional RSS comparisons are not sufficient enough to make a vertical handover decision, as they do not consider the various available options for the mobile user. Other factors, such as monetary cost, network conditions, mobile node conditions, user preferences and so on, must be considered, as well as the capabilities of the various networks in the vicinity of the user. Thus, a more sophisticated, adaptive and intelligent approach is needed to implement the vertical handover mechanisms to produce a satisfactory result for both the user and the network. In addition, services can be composed of applications that could use different network interfaces. A single connection session for the specific service will be maintained efficiently by the service control. Therefore, a thorough evaluation of the types of services and applications are necessary in making a proper decision on the network interface.

In this paper, we propose the Autonomic Handover Manager (AHM) based on the autonomic computing concept [3,4] to determine the best network interface to handover in $4 \mathrm{G}$ networks. This manager generates a policy to determine the best network interface for the specific service by using the context from the terminal, the network and the user. Then, it maintains by itself to provide seamless service to the user with the self-management mechanism.

The remainder of this paper is organized as follows. Section 2 describes the related work on the handover decision models, context management, and the autonomic computing concept. Section 3 explains our solution approach. Section 4 presents the architecture of AHM and the context evaluation function. Section 5 presents an interesting scenario using our proposed model and evaluates it qualitatively as the user-centric aspect. Finally, conclusions are drawn and future work is discussed in Section 6.

\section{Related Work}

In this section, we present the previous work and the problems related to the handover decision model. We also describe the context management in mobile environments for the vertical handover and the concept of autonomic computing.

\subsection{Handover Decision Models}

Related work on the handover decision model has been presented in recent research literature. A policy-based handover scheme has been proposed in [5], where the authors designed a cost function to decide the "best" moment and interface for the vertical handover. However, the cost function presented in that paper is very preliminary and cannot handle sophisticated configurations. The logarithmic function used in the cost function also has difficulty in representing the cost value while the 
value of the constraint factor is zero (e.g., the connection is free of charge). Another scheme proposed in [6] models the handover with HTTP traffic, but it may have problems with other types of traffic, such as video and audio streaming, where the bandwidth demand is much higher than HTTP traffic. A smart decision model in [7] is proposed to perform vertical handover to the "best" network interface at the "best" moment and is tested on the Universal Seamless Handover Architecture (USHA) [8]. The smart decision model is based on the properties of available network interfaces (link capacity, power consumption and link cost), system information (remaining battery) and user preferences. Although the model presented a detailed example on the USHA test-bed, it was not enough to describe how to achieve the properties and the meaning of cost value. The authors of [9] aim to understand how to define a metric in order to devise a solution that balances the overall cost of the vertical handovers with the actual benefits they bring to the user's networking needs. This way, each mobile user could autonomously apply the handover decision policy, which is more convenient to the user's specific needs. A vertical handover decision function proposed in [10] allows the user to strategically prioritize the different network characteristics such as network performance, user preference and monetary cost. This function is simple and can be easily applied to any vertical handover approach. The authors presented some characteristics for decision function such as the cost of service, security, power requirements, proactive handover, quality of service and velocity. However, this study was not enough to describe how to define each characteristic and to present the example to validate the decision function. Also, it did not consider the cost function in terms of the service or the application.

\subsection{Context Management}

The context management framework proposed in [11] has optimized the services in the mobile environment based on the context information. This ensured that the correct context information is available at the right place at the right time, and that it handles diverse, dynamic and distributed context information. This was implemented in the prototype [12], where different context formats and exchange algorithms were evaluated in detail. The context information can be applied to the decision model for vertical handover. In general, context information can be static or dynamic and can come from different network locations, protocol layers and device entities.

Table 1. Context Information Classification

\begin{tabular}{|l|l|l|}
\hline \multirow{3}{*}{ Static } & Mobile Devices & Network \\
\cline { 2 - 3 } & User settings and profile & User profile and history \\
\cline { 2 - 3 } & Application setting & Network location, capabilities and services \\
\cline { 2 - 3 } Static in a cell & Willingness to pay & Charging models \\
\hline \multirow{2}{*}{ Dynamic } & Type of application & Potential next access point \\
\cline { 2 - 3 } & Application requirements & Location information and location prediction \\
\cline { 2 - 3 } & Device status (battery, interface status) & Network status such as signal strength \\
\hline
\end{tabular}

Table 1 [12] provides a classification of context information to be considered in the mobile environments. This table is clearly just a snapshot, as other types of context 
information may be considered as well. They presented their context management framework which can collect and process the relevant context information using context repositories, context server, mobile context client and network traffic monitor. They compiled the context information into a decision matrix exploiting context information for the horizontal handover. We have presented the mechanism to apply the context information for the vertical handover more complicated than the horizontal handover.

\subsection{Autonomic Computing}

Autonomic computing was proposed as a systematic approach to achieving computerbased systems managing themselves without human interventions [3]. An autonomic computing system has four basic characteristics for self-management. They are selfconfiguring, self-healing, self-optimizing and self-protecting: Self-configuration frees people to adjust properties of the system according to changes of the system and environment; self-healing frees people to discover and recover or prevent system failures; self-optimization frees people to achieve best-of-the-breed utilization of resources; self-protection frees people to secure the system.

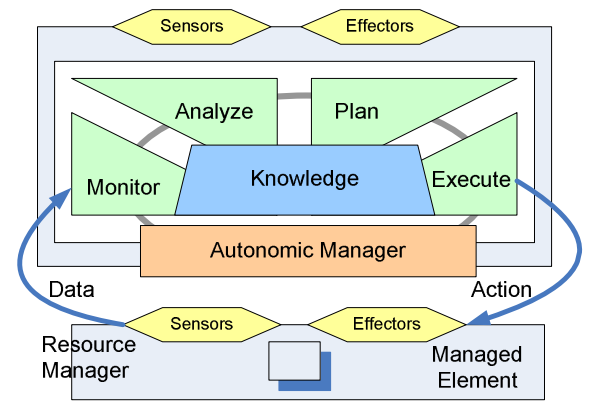

Fig. 1. The Architecture of Autonomic Manager

An autonomic manager is an implementation that automates some management function and externalizes this function according to the behavior defined by management interfaces. As shown in Fig 1, the architecture dissects the loop into four parts that share knowledge. These four parts work together to provide the control loop functionality.

- Monitor function provides the mechanisms that collect, aggregate and filter and report details collected from a managed resource.

- Analyze function provides the mechanisms that correlate and model complex situation. These mechanisms allow the autonomic manager to learn about the IT environment and help predict future situations.

- Plan function provides the mechanisms that construct the actions needed to achieve goals and objectives. The planning mechanism uses policy information to guide its work

- Execute function provides the mechanisms that control the execution of a plan with considerations for dynamic updates. 
These four parts communicate and collaborate with one another and exchange appropriate knowledge and data to achieve autonomic management.

\section{Solution Approach}

We propose a handover decision model using the context information to overcome the limitations of the previously proposed decision models in Section 2. In the mobility management, the decision function to decide a handover is one of the most important functions. It should use the context information of the network, the mobile terminal and the user.

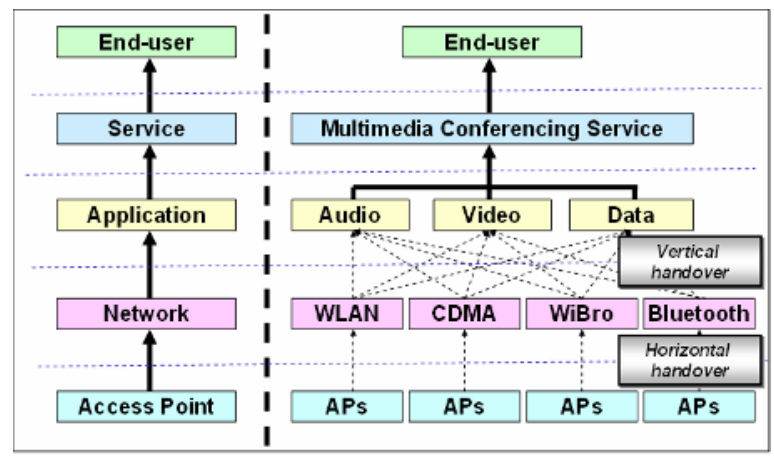

Fig. 2. Layering for general service and multimedia conferencing service

Fig 2 shows the general service scenario on the left and multimedia conferencing service on the right considering the application, network and access point in a heterogeneous mobile network environment. For instance, the end-user is using the multimedia conferencing service on the mobile terminal which can access multiple radio networks using multiple network interfaces. It is composed of multiple applications such as audio, video and data application. Each application succeeds in making a session with one of multiple access networks such as WLAN, CDMA, WiBro and Bluetooth. Then, the mobile terminal connects with one of the access points. There are two kinds of handover; the vertical handover which occurs when a different type of access network is selected, and the horizontal handover which occurs when another access point is selected within the same type of access network. As mentioned earlier, traditionally, the selections of access network and access point are based on the signal strength. In future, more parameters related to QoS, battery and service pricing should be considered because the end-users might use the service according to their needs which could prevent them from being interested in selections of access network and access point.

In order to solve such problems, we propose an autonomic handover manager to make a decision to handover with the context information. The autonomic handover manager is one of the key components which compose the terminal management system. It can monitor, analyze, plan and execute using sensors and effectors. It needs the context evaluation function to choose a policy, using the context information. The 
context evaluation is represented by the context evaluation matrix accompanied by additional rules and policies. The context information is provided by the context management framework which supports various context repositories distributed in the networks. The context server collects the relevant context information from the context repositories and prepares the evaluation matrix. The mobile terminal evaluates the evaluation matrix for context processing and makes a decision on handover. In the next section, we describe our solution by the approach mentioned above.

\section{Autonomic Handover Manager}

In this section, we present the autonomic handover manager (AHM) using the context information of the terminal, the network and the user based on the autonomic computing concept. AHM is one of the core components in the terminal management system (TMS). The purpose of AHM is to provide a decision for selecting the best network interface and the best AP using the context information autonomously. The main idea of AHM is to consider not only the signal strength but also the context information to select the network interface. We describe the architecture of AHM and the context evaluation function.

\subsection{Architecture}

Fig 3 illustrates the architecture of the TMS considering the context server. Traditionally, TMS selects the network interface for vertical handover by the signal strength only. However, TMS for $4 \mathrm{G}$ networks requires an innovative architecture that is capable of dynamically selecting the appropriate network through which services can be obtained efficiently in terms of cost and QoS in a transparent manner. Our proposed architecture which has the AHM is able to use the context information from the mobile node and the context server. The context server collects the network context information from the respective operations and support systems (OSS). Currently, this is not realistic because it is difficult and rather impossible to share the information among the service providers. We assume that this will be possible in a $4 \mathrm{G}$ network and service environment.

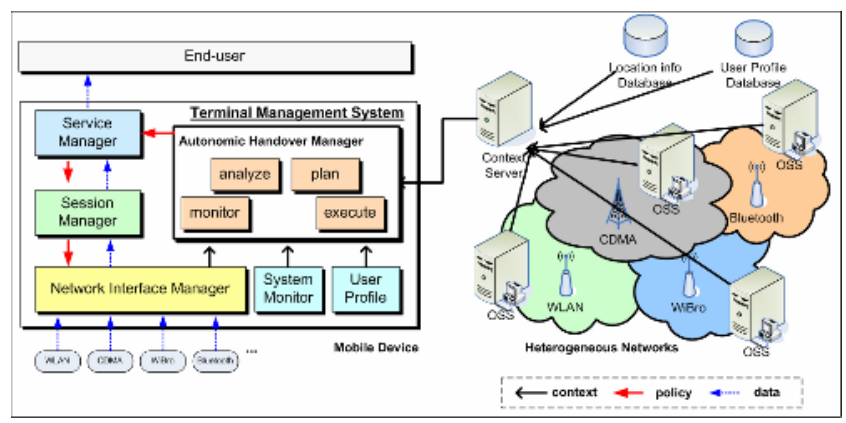

Fig. 3. Architecture of Terminal Management System using Autonomic Handover Manager 
There are two major parts in this architecture; network side and terminal side. An optimal handover decision is to be achieved through their joint contributions. On the network side, the OSS of each network will perform network monitoring and report to the context server. The various repositories distributed in the networks will store the context information such as location information and user profiles. The context server located in the network collects the relevant context information from the context repositories. It prepares the evaluation matrix in response to the handover decision request from TMS. On the terminal side, the TMS will interact with the context server mentioned above for the purpose of making the optimal selection of the appropriate radio segment to which the terminal will eventually be assigned. The terminal's estimation of signal strength and QoS levels in the system are beneficially combined for making an informed selection of the appropriate radio technologies through which services can be obtained as efficiently as possible. Thus, both the network and the terminal contribute useful information towards the optimal decision.

AHM interacts with context server, system monitor and user profile repository. After processing the context evaluation, it decides on an appropriate policy and informs it to the service manager. The service manager manages the mapping of service and applications. It creates a session for each application using the policy from the AHM. The session manager maintains the created session and sends the decision of the appropriate network interface for the application to the network interface manager. The network interface manager serves two purposes. One is the retrieval of measurements at L2 level in the network interface and the other is the connection of the appropriate interface during a handover. Our management solution is located in the management layer and is independent of the OSI layers. Thus, it can control and monitor all layers. The system monitor collects the system information such as the remaining quantity of the battery and the memory. There are user settings on the services in the user profile.

AHM provides four major functions to decide an appropriate policy by the context information as mentioned in Section 2.

- Monitor function provides the mechanisms that collect, aggregate and filter the dynamic context from the terminal such as the received signal strength, cpu usage and remained battery.

- Analyze function provides the mechanisms that correlate and model complex situation. This updates the evaluation matrix from the context server by the monitored dynamic context.

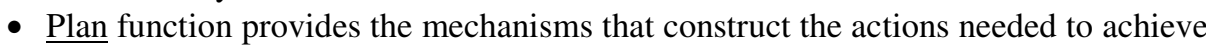
goals and objectives. This uses policy information to guide the result of evaluating matrix.

- Execute function provides the mechanisms that control the execution of a plan with considerations for dynamic updates. This publishes the policy to the service manager.

These four parts work together to provide the control loop functionality. The four parts communicate and collaborate with one another and exchange appropriate knowledge and data. 


\subsection{Context Evaluation Function}

We show the context evaluation function to make optimal handover decisions based on the context information. More specifically, we need to select an appropriate network interface for each application considering the changing context information. This function requires a flexible data structure to express both user preferences and operator preferences. Thus, we define the context evaluation matrix which is flexible for updating.

Eq 1 shows the evaluation matrix for a service. A service is composed of $m$ applications and the mobile terminal has $n$ network interfaces. The k parameters are considered as well.

$$
\begin{aligned}
& \left(\begin{array}{cccc}
S_{11} & S_{12} & \cdots & S_{1 n} \\
S_{21} & S_{22} & \cdots & S_{2 n} \\
\vdots & \vdots & \ddots & \vdots \\
S_{m 1} & S_{m 2} & \cdots & S_{m n}
\end{array}\right)=\left(\begin{array}{cccc}
C_{11} & C_{12} & \cdots & C_{1 k} \\
C_{21} & C_{22} & \cdots & C_{2 n} \\
\vdots & \vdots & \ddots & \vdots \\
C_{m 1} & C_{m 2} & \cdots & C_{m k}
\end{array}\right) \otimes\left(\begin{array}{cccc}
R_{11} & R_{12} & \cdots & R_{1 n} \\
R_{21} & R_{22} & \cdots & R_{2 n} \\
\vdots & \vdots & \ddots & \vdots \\
R_{k 1} & R_{k 2} & \cdots & R_{k n}
\end{array}\right) \\
& \Rightarrow\left(\begin{array}{llll}
C_{i 1} & C_{i 2} & \cdots & C_{i k}
\end{array}\right) \otimes\left(\begin{array}{llll}
R_{1 j} & R_{2 j} & \cdots & R_{k j}
\end{array}\right)^{T}=f\left(C_{i 1} R_{1 j}, C_{i 2} R_{2 j}, \cdots, C_{i k} R_{k j}\right) \\
& \left(0 \leq C_{i j} \leq 1,0 \leq R_{i j} \leq 1\right)
\end{aligned}
$$

Eq. 1. Context evaluation matrix ( $m$ : \# of applications, $k$ : \# of parameters, $n$ : \# of network interfaces)

- $S_{i j}$ : The expected value of $i$ application on using $j$ network interface $(i$ : index of application, $j$ : index of network interface)

- $C_{i j}$ : Coefficient (weight) of different parameters. This can be service specific (e.g. user preference) or service and operator specific (e.g., pricing) ( $i$ : index of application, $j$ : index of parameter)

- $R_{i j}$ : Parameters which will affect the decision (e.g., available bandwidth, signal strength ) ( $i$ : index of parameter, $j$ : index of network interface)

In addition to the matrix, additional rules or conditions are considered which assign values to parameters. For instance, there are some rules to set the upper or lower bounds. These rules have to be evaluated before evaluating the matrix. The $C$ matrix is set by the user preferences. We propose a score function of $f\left(C_{i 1} R_{1 j}, C_{i 2} R_{2 j}, \cdots, C_{i k} R_{k j}\right)$. This function should present the effective score for evaluation. For instance, it might be the SUM function as shown in Eq 2.

$$
f\left(C_{i 1} R_{1 j}, C_{i 2} R_{2 j}, \cdots, C_{i k} R_{k j}\right)=\sum_{m=1}^{k} C_{i m} R_{m j}
$$

Eq. 2. The example of the score function, SUM function

As mentioned above, $\mathrm{C}$ matrix is a combination of applications and parameters and $\mathrm{R}$ matrix is a combination of parameters and networks. For example, let us consider the multimedia conferencing service (MCS), which is composed of audio application (A1), video application (A2) and data application (A3). The mobile terminal is able to 
access multiple networks such as CDMA (N1), WiBro (N2), IEEE 802.11b WLAN (N3), and Bluetooth (N4). In addition, we can consider context information, such as, available bandwidth, power consumption, signal strength, cost of service, velocity, QoS and so on. The parameters are defined by the combination of such context information. We define the parameters as Quality (P1), Price (P2), Velocity (P3) and Power (P4). The values of $\mathrm{A}$ and $\mathrm{P}$ are set by the user preferences. $\mathrm{P} 1 / \mathrm{N}$ is made by available bandwidth, signal to noise ratio (SNR), bit error rate (BER) and class of quality. $\mathrm{P} 2 / \mathrm{N}$ is made by cost of service, billing mechanism and class of quality. $\mathrm{P} 3 / \mathrm{N}$ is made by the supported moving speed. $\mathrm{P} 4 / \mathrm{N}$ is made by signal strength, CPU usage and LCD refresh rate. We can make $\mathrm{R}$ matrix $(\mathrm{P} \times \mathrm{N})$. It emphasizes that the parameter is an important factor in the network. Then, we can make C matrix (A x P). It presents the weight of the parameter in the specific application.

After achieving the complete matrix of $\mathrm{C}$ and $\mathrm{R}$, we can achieve $\mathrm{S}$ matrix. Eq 3 shows that we can obtain the map of application and network interface applying policies.

$$
\left(\begin{array}{cccc}
S_{11} & S_{12} & \cdots & S_{1 n} \\
S_{21} & S_{22} & \cdots & S_{2 n} \\
\vdots & \vdots & \ddots & \vdots \\
S_{m 1} & S_{m 2} & \cdots & S_{m n}
\end{array}\right) \stackrel{\text { policies }}{\longrightarrow}\left(\begin{array}{c}
S_{1 j} \\
S_{2 k} \\
\vdots \\
S_{m l}
\end{array}\right) \equiv\left(\begin{array}{c}
A_{1}: N_{j} \\
A_{2}: N_{k} \\
\vdots \\
A_{m}: N_{l}
\end{array}\right) \rightarrow Q_{i}
$$

Eq. 3. The map of application and network interface by the evaluation matrix ( $m$ : \# of applications, $n$ : \# of network interfaces, $i$ : index of services )

- $S_{i j}$ : result of a service ( $i$ : index of application, $j$ : index of network interface)

- $A_{i}$ : application (e.g., Audio, Video, Data ) (i: index of application)

- $N_{i}$ : network interface (e.g., WLAN, CDMA, WiBro, Bluetooth) ( $i$ : index of network interface)

- $Q_{i}$ : Service (e.g., Multimedia conferencing service)

As a result of context processing, we can achieve $\boldsymbol{Q}$ matrix for all of services. We will describe the detailed example of the context evaluation matrix in the next section using a sample scenario.

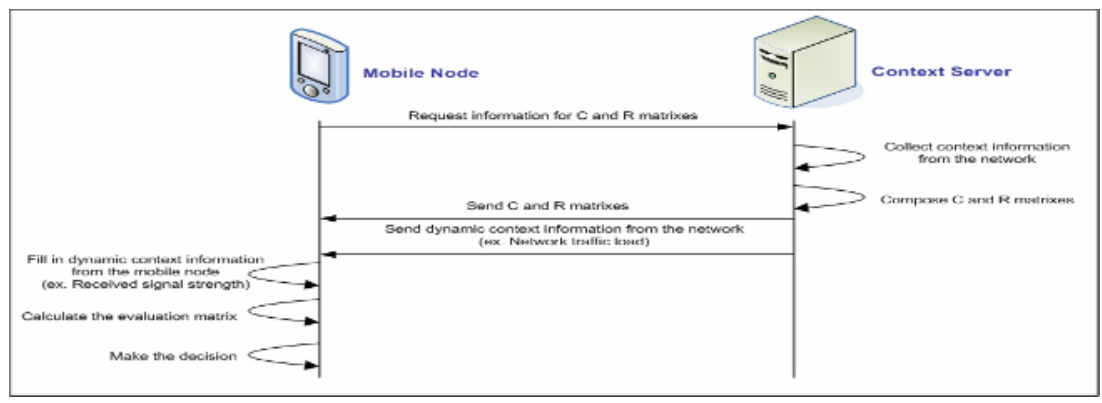

Fig. 4. Context processing between the mobile node and the context server 
Fig 4 shows the procedure for context information collection and processing between the mobile node and the context server. The mobile node sends a request to the context server to ask for an evaluation matrix. The context server collects the context information from the network and composes an evaluation matrix based on the received static context information from the mobile node as shown in Table 1. It fills in the known values of the parameters which can be drawn from the static context information in the network. Then, it sends the evaluation matrix to the mobile node and also sends updates of the parameters for dynamic context information in the network to the mobile node. The mobile node fills in the dynamic context information and calculates the evaluation matrix when a decision is needed. At this time, the context server should send the evaluation matrix when the dimension or component of the matrix is changed. Finally, the mobile node makes the decision based on the result matrix $\boldsymbol{Q}$. A vertical handover is usually needed while the terminal is moving. Thus, the handover decision must be made very quickly within the specified time. Otherwise, the terminal will not be able to handover to the optimal network to provide continuous and seamless service.

\section{Evaluation}

In this section, we present an interesting scenario in $4 \mathrm{G}$ networks along with the evaluation result using our AHM to manage handovers with the goal of optimizing the selection of the new access network by the context information from the mobile terminal, the network and the user.

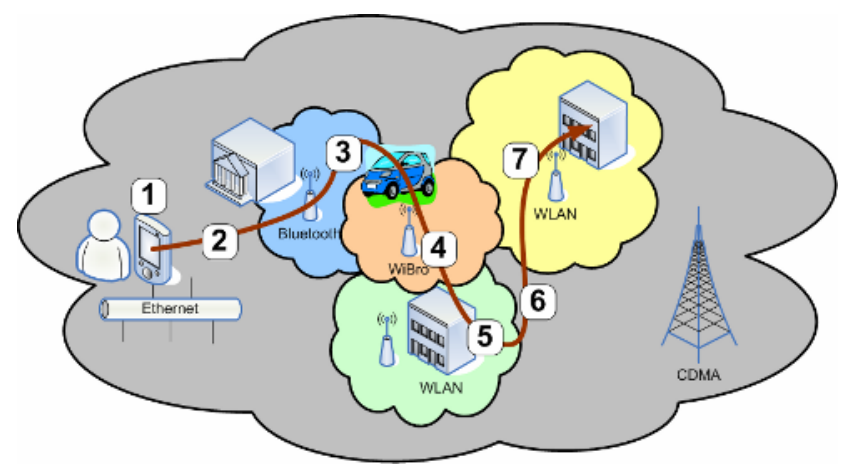

Fig. 5. Evaluation scenario (using multimedia conferencing service in $4 \mathrm{G}$ networks)

A business man is using the multimedia conferencing service on his PDA with a colleague in another city. He is initially connected from his home as in Fig 5 . He prefers to use the service that is cost-effective, battery-effective and quality-effective. In order to satisfy his needs, TMS should decide the best network interface for him to use. AHM is able to provide the solution by evaluating the context information. The user can set the preferences of the application on cost, power and quality. After setting them once, the user does not wish to set them again while using the service. 
Table 2. The result of AHM evaluation

\begin{tabular}{|l|l|l|l|l|l|l|c|}
\hline \multicolumn{1}{|c|}{ APP } & \multicolumn{1}{c|}{$\mathbf{2}$} & \multicolumn{1}{c|}{$\mathbf{2}$} & \multicolumn{1}{c|}{$\mathbf{3}$} & \multicolumn{1}{c|}{$\mathbf{5}$} & $\mathbf{6}$ & $\mathbf{7}$ \\
\hline $\begin{array}{l}\text { Available } \\
\text { Networks }\end{array}$ & $\begin{array}{l}\text { CDMA } \\
\text { Wired }\end{array}$ & CDMA & $\begin{array}{l}\text { CDMA } \\
\text { Bluetooth }\end{array}$ & $\begin{array}{l}\text { CDMA } \\
\text { WiBro } \\
\text { WLAN } \\
\text { Bluetooth }\end{array}$ & $\begin{array}{l}\text { CDMA } \\
\text { WLAN }\end{array}$ & CDMA & CDMA \\
WLAN \\
\hline Audio & Wired & CDMA & Bluetooth & WiBro & CDMA & CDMA & WLAN \\
\hline Video & Wired & X & Bluetooth & WiBro & WLAN & X & WLAN \\
\hline Data & Wired & CDMA & Bluetooth & WiBro & WLAN & CDMA & WLAN \\
\hline
\end{tabular}

In Section 4, we have already explained the context evaluation matrix of the multimedia conferencing service. We have applied it to the above scenario. In Fig 5, we are able to make a decision for the vertical handover in $3,4,5$ and 7 . The user prefers to use the best quality audio application and video application and data application at the cheapest price. Table 2 shows the result of AHM evaluation for the vertical handover.

Even though there are same network choices in 5 and 7 , the result is different because the network traffic load of 5 might be heavier than 7 . We describe the process of the evaluation about 5 in Fig 5. In the 5th case, the available networks are WLAN and CDMA. The parameters are only quality and cost. First of all, AHM should make the context evaluation matrix by collecting the context information from the terminal and the network.

$$
\begin{aligned}
& C:(\text { Audio,Video, Data }) \times(\text { Quality, Cost }), R:(\text { Quality }, \text { Cost }) \times(\text { WLAN }, \text { CDMA }) \\
& f\left(C_{i 1} R_{1 j}, C_{i 2} R_{2 j}, \cdots, C_{i k} R_{k j}\right)=\sum_{m=1}^{k} C_{i m} R_{m j} \\
& \left(\begin{array}{cc}
0.9 & 0.1 \\
0 & 1.0 \\
0.1 & 0.9
\end{array}\right) \otimes\left(\begin{array}{ll}
0.3 & 0.5 \\
0.7 & 0.3
\end{array}\right)=\left(\begin{array}{cc}
0.34 & 0.48 \\
0.7 & 0.3 \\
0.66 & 0.32
\end{array}\right) \stackrel{p}{\longrightarrow}\left(\begin{array}{c}
\text { Audio }: \text { CDMA } \\
\text { Video }: \text { WLAN } \\
\text { Data }: \text { WLAN }
\end{array}\right)
\end{aligned}
$$

Eq. 4. The example of the context evaluation matrix

In Eq 4, the quality value of WLAN is 0.3 while that of CDMA is 0.5 . This means that CDMA has a better quality because the network traffic load might be heavy in WLAN. Moreover, the result matrix shows that the audio application should select CDMA as the best network in this situation because the result of CDMA is greater than that of WLAN. In contrast, Video and Data applications should select WLAN. In this case, the calculation method of the normalized value of the matrix is an important aspect to be evaluated. However, this is not a concern for this paper. We assume that the other components of the terminal management system can explore this area.

\section{Conclusion and Future Work}

The handover management in $4 \mathrm{G}$ networks is a very important issue. We need a clever and seamless vertical handover mechanism when moving among various 
access networks. In this paper, we have proposed the AHM to provide a solution for determining the best network interface for the service. The decision is made by using the context information from the mobile node, networks and the user as well as the received signal strength. Thus, the end-users can enjoy efficient services according to their preferences in $4 \mathrm{G}$ networks. $\mathrm{AHM}$ is based on the autonomic computing concept. It provides a good policy for the vertical handover using the context information without user's interventions. We have presented the static and dynamic context information of the mobile node and the network. AHM has four major functions such as monitoring, analyzing, planning and executing. We have also described how to compose the context evaluation function and formulate a policy.

We have presented an interesting scenario in $4 \mathrm{G}$ networks to validate our approach, which is to use the multimedia conferencing service on the mobile terminal through the heterogeneous networks. The result has shown that the users are able to use flexible services according to their preferences on cost-effective policy, qualityeffective policy and battery-effective policy.

For future work, we will present more concrete context information and improve the AHM by considering it and optimizing the context evaluation function. And, we will present more exact values for the evaluation matrix and analyze the overhead and cost associated with setting up the context server and transmitting the evaluation matrix periodically. Then, we will implement it on the open mobile platform and validate the feasibility. We will present how to apply the user preferences to the coefficient matrix of the context evaluation function easily and study the context management in 4G networks. Finally, we will test the various scenarios using the user profile data and network traffic analysis data to present the efficiency of our AHM.

\section{References}

[1] S. Balasubramaniam and J. Indulska, "Vertical Hanover Supporting Pervasive Computing in Future Wireless Networks," Computer Communication Journal, Special Issue on 4G/Future Wireless Networks, vol 27/8, pp. 708-719, 2003.

[2] E. Gustafsson and A. Jonsson, “Always Best Connected," IEEE Wireless Communications, vol 10, pp. 49-55, February 2003.

[3] IBM Corporation: An architectural blueprint for autonomic computing. White Paper, (2003).

[4] J.O. Kephart and D.M. Chess, "The Vision of Autonomic Computing," Computer, vol. 36, no. 1, pp. 41-52, Jan. 2003.

[5] H.J. Wang, R.H. Katz, and J. Giese, "Policy-Enabled Handoffs Across Heterogeneous Wireless Networks," Proc. of ACM WMCSA, 1999, p. 51.

[6] M. Angermann and J. Kammann, "Cost Metrics For Decision Problem In Wireless Ad Hoc Networking," in Proceedings IEEE CAS Workshop on Wireless Communications and Networking, (Pasadena, USA), September 2002.

[7] Chen L. -J., Sun T., Chen B., Rajendran V., and Gerla M., "A Smart Decision Model for Vertical Hanoff," In Proceedings 4th ANWIRE International Workshop on Wireless Internet and Research (ANWIRE 2004), Athens, Greece, 2004.

[8] Chen L. -J., Sun T., Chen B., Rajendran V., and Gerla M., "Universal Seamless Handoff Architecture in Wireless Overlay Networks," Technical Report 040012, UCLA CSD, 2004 
[9] A. Calvagna and G. Di Modica, "A User-Centric Analysis of Vertical Handovers," ACM Wireless Mobile Applications And Services On WLAN Hotspots 2004(WMASH 2004), Philadelphia, PA, USA, pp. 137-146, 2004.

[10] A. Hasswa, N. Nasser and H.S. Hassanein, "Generic Vertical Handoff Decision Function for Heterogeneous Wireless Networks," IFIP Conference on Wireless and Optical Communications, pp. 239-243, March 2005.

[11] Q. Wei, C. Prehofer, "Context Management in Mobile Environment," In Proceedings 3th ANWIRE International Workshop on Wireless Internet and Research (ANWIRE 2003), November 13, Paris, France, 2003.

[12] Paulo Mendes, Christian Prehofer, Qing Wei, "Context Management with Programmable Mobile Networks," IEEE Computer Communications Workshop, October 20-21, Dana Point, California, USA, pp. 217-223, Oct. 2003. 